\title{
A Repository Collaboration Model to Gamify Education Using Synergistic Digital Hub
}

\author{
Alaa Saggah \\ School of Computing and Digital \\ Technologies Staffordshire University \\ Stoke on Trent, ST4 2DE, UK \\ Makkah Community College \\ Umm Al-Qura University \\ Kingdom of Saudi Arabia \\ ORCID (0000-0001-6650-2028)
}

\author{
Anthony S Atkins \\ School of Computing and Digital \\ Technologies, Staffordshire University \\ Stoke on Trent, ST4 2DE, UK \\ a.s.atkins@staffs.ac.uk
}

\author{
Russell J Campion \\ School of Computing and Digital \\ Technologies, Staffordshire University \\ Stoke on Trent, ST4 2DE, UK \\ r.j.campion@staffs.ac.uk
}

\begin{abstract}
This paper discusses the benefits of applying gamification elements to enhance pupils' motivation and engagement towards learning. During the COVID-19 lockdown there has been a drastic change in school learning paths which have led to home schooling practice. While there have been some attempts to overcome the challenges of changed learning methods, there are some opportunities for further innovation. The aim of the proposed Synergistic Digital Hub (SDH) is to connect all stakeholders and bring like-minded people to build a repository that represents the best practice to deliver learning objectives in a distance learning environment.
\end{abstract}

Keywords-Gamification, distance learning, home schooling, COVID-19, motivation, Synergistic Digital Hub

\section{INTRODUCTION}

The current uncertain time of the lockdown due to COVID-19 has resulted in providing online alternative(s) to support home schooling. The use of emerging technologies to promote learning such as mobile technologies and online courses is always an interesting research area. Gamification elements such as rewards, learderboards and social engagement have been used by teachers as part of in-class activities to reinforce learning objectives. The incorporation of electronic games in teaching and learning from literature reviews has been shown to be beneficial [1] and [2]. In this paper we propose a Synergistic Digital Hub (SDH), a model to support a gamified home schooling environment. The model manages the integration of different parent's expertise in a home schooling environment and educators learning views to incorporate gamification elements. The intention is to set up repository so that educational games are accessible to parents in both electronic and non-electronic format.

The challenge of COVID-19 lockdown was acknowledged as a global pandemic overnight in many countries and the underestimation of that threat has led to unprecedented measures. In many cases that includes school closure which poses an obstacle in pupil's learning paths. This paper reviews some of the initiatives in the UK to build on the achievements and overcome the challenges represented in BBC bitesize Daily lessons [3] etc. This paper suggests a model to provide a route map for development of educational games to support the alternative pathway initiatives of learning and connecting stakeholders.

This paper is composed as follows; Section 2: outlines gamification and its use to enhance engagement and motivation. 3: discusses a review of some current efforts to support home schooling followed by Section 4 which outlines a proposed model in this paper to support all involved parties. Finally, Section 5 outlines the conclusion to the paper.

\section{GAMifiCATION RELATION TO ENGAGEMENT AND MOTIVATION}

Many studies have discussed the benefits of gamification as a motivation boost. Some studies specifically suggest the use of rewards in many forms such as learderboards, points, badges, etc... which will help maintain the students' interested in playing educational games and therefore, enhance the educational experience [4], [5], [6] and [7]. Other studies such as [8] and [2] discussed the positive effect of gamification elements in students' engagement and enjoyment in the courses. More comprehensive studies, which have been applied to University courses, indicated that gamification has enhanced students' motivation, attention, engagement and learning performance [1].

Gamification can be applied through various elements For instance, reference [5] suggests to include Empowerment of creativity \& feedback and Ownership. Another study suggests progressive goals, social engagement, timing, rewards and progress presentation [9].

\section{REVIEW OF SOME CURRENT EFFORTS TO SUPPORT HOME SCHOOLING}

This section provides a few examples of the current practices to provide an alternative pathway of learning. Firstly, daily lessons provided by $\mathrm{BBC}$ Bitesize. BBC is a public service broadcaster in the United Kingdom. In an attempt to support home schooling BBC Bitesize webpage offers support as indicated:- "Bitesize guides are written by teachers and subject experts and are mapped to follow the curricula in the UK." [3]. There are daily updates of 3 new lessons that include all school years, as illustrated in Figure 1

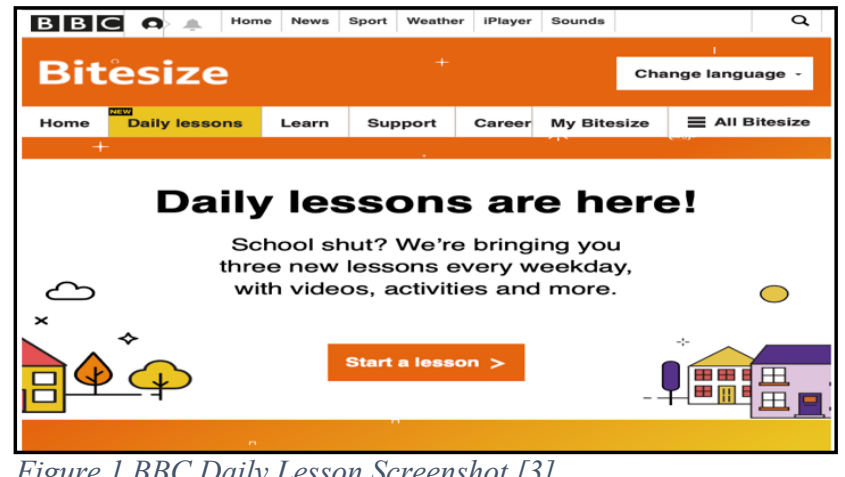

Figure 1 BBC Daily Lesson Screenshot [3] 
The lesson includes textual information outlining the lesson and instructions, together with a short video of parts of the lesson, printable games and sometimes an interactive game. The website works as a distribution platform and there is no communication or feedback given to parents and students. For example, there is no students profile and consequently, no personalization or record of individual progress on the website. Adding progress information for individuals is considered a gamification element that provides a sense of accomplishment [5]. Another example of current practices to overcome school closure is English with Holy channel on YouTube [10] for broadcasting lessons to support learning during lockdown. Holy provides English lessons to support Key Stage 2 students. There are some schools that decided to continue to deliver virtual classes experience using video conferences application while other stayed on weekly email activities sheets. All efforts may be appreciated by parents considering the abrupt nature of schools' closure; however, they sometimes lack the motivation for students which can be met through gamifying the learning environment for the pupils.

\section{THE PROPOSED MODEL}

In this paper we suggest a Synergistic Digital Hub (SDH), illustrated in Figure 2, that facilitates a collaboration platform that combines expertise of all involved parties to support home learning by creating a community that includes teachers, parents, students, and game developers and government body representation. Uniting the effort helps to create a community where support is found, and collaboration is facilitated. This also enhances the ability to transform a game from a pencil and paper into an electronic format using an iPad App and vice versa. Furthermore, incorporating with an outdoor activity, especially in the current situation of lockdown, for example, playing a maths game about fractions, in the traditional class the pupils will be using crayons to colour parts of a shape to understand the fraction resemblance, whereas in an iPad version, they would use a paint bucket on the screen. Both settings of the game would represent the same learning objective.

Similarly, looking at a path in a park that a family walk through and using that to perform a simple maths task of counting steps. The strength would be in a family sharing their outdoor maths activity that other parents could adopt with their families and provide feedback. Feedback might include a suggestion to motivate a child of a certain age.

Furthermore, teachers might suggest a new maths task building the learning progression of the outdoor activity. Another benefit would be a game developer transforming the game into an App as another learning medium. Then, the government body would decide if the game is worth investing the financial and human resources based on the feedback and the interaction the game would initially have across all stakeholders on the platform; students, parents, teachers etc.

Another innovation would be bringing like-minded people to build a repository that represents the best practice to deliver learning objectives in a distance learning environment. Initiatives from the Ministry of Education would need to be implemented to filter and edit ideas from a pedagogical perception. Also, these initiatives could administer financial bonus, secondments, badges and certificates for participating teachers to develop the repository. This environment could work as an extension of traditional learning classes but also, could function independently in situations such as the COVID-19 lockdown and [11] acknowledged the positive impact gamification had on students' learning progression, represented by play-based activities such as craft and puzzles. The functional aspects depicted in Figure 2 are outlined as follows:

\section{A. Repository/collaboration platform}

In this model the platform will facilitate collaboration amongst all stakeholders; students, parents, teachers, game developers and Government bodies. The learning activities can be categorized based on learning theories stipulating the following features;

- A repository of gamified learning examples

- Personal profile of users

- Log achievements of students

- Suggest activities for students based on their learning style and personal preferences

\section{B. Government body}

In this model we would suggest the following features to be included;

- Endorsement

- Financial resources

C. Game Developers

Game developers' expertise would benefit in taking an educational game into an electronic form. Their collaboration with teachers has been discussed in [12] [13]. In this model we suggest the following features;

- $\quad$ Digitalise the game ideas

- $\quad$ Prototype

Furthermore, game developers should be able to apply for financial initiatives required to support the electronic game design team from Government resources.

\section{Pupils}

The aim is to promote pupils' motivation towards learning and gamification elements that would enhance details discussed in Section 2. In this model we suggest the following features to be included;

- $\quad$ Play the game

- Social interaction

- Personalization

- Rewards

- Rate the game

\section{E. Parents/carers}

Including parents as part of educational process will lead to useful cooperation experience [14] and in this model we suggest the following features;

- $\quad$ Suggest new ideas

- Evaluate children's motivation on available games 


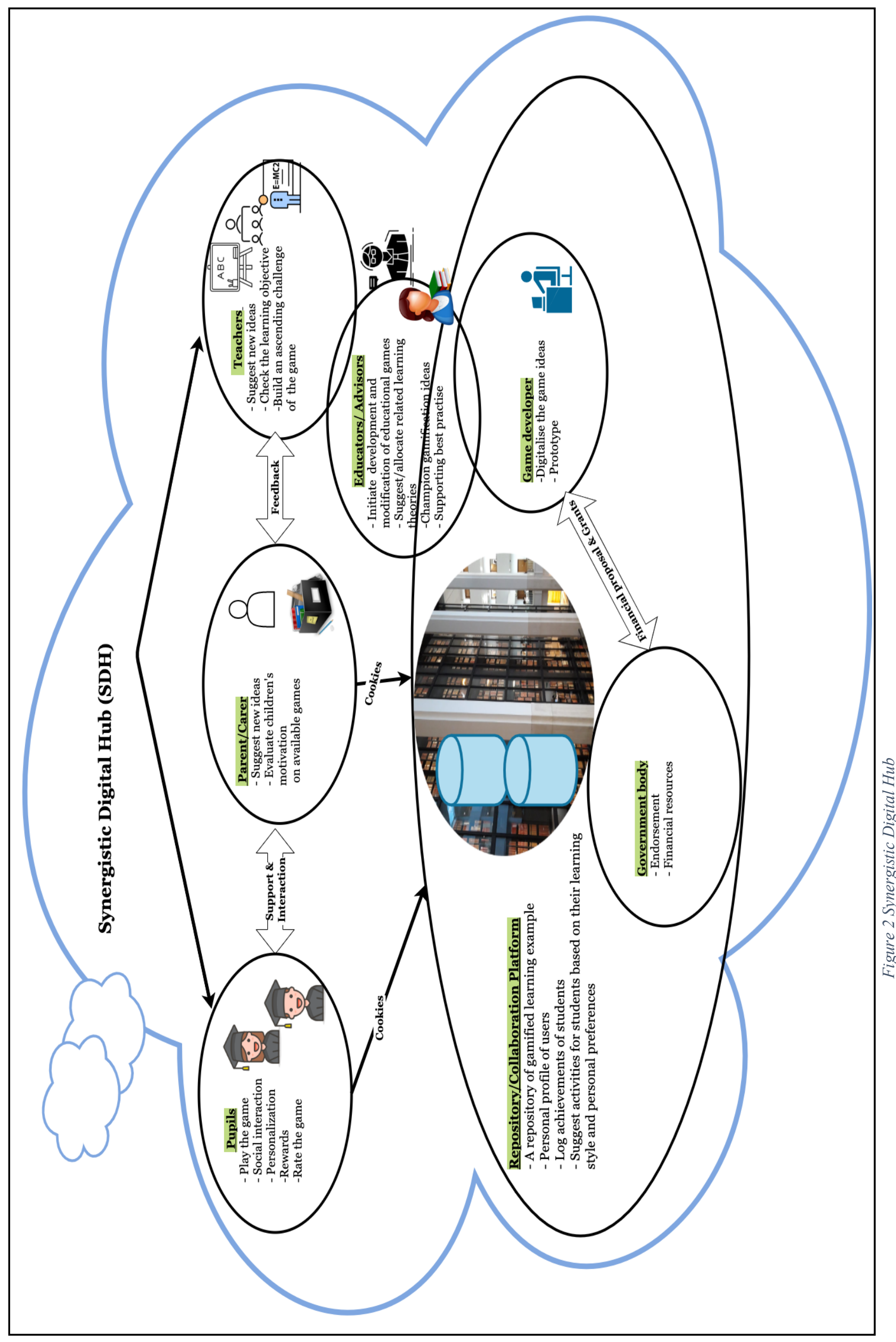


Furthermore, the parents/carers should be encouraged to support and interact with their children in using the platform.

\section{F. Teachers}

Teachers' role has been as an important part of gamification in education in identifying goals or introducing the game to students, discussed in [8], [15], [16], [17], [18], [19], [20] and [21]. In this model we suggest the following features to be included;

- $\quad$ Suggest new ideas

- Check the learning objective

- Build an ascending challenge of the game

\section{G. Education advisor}

- In this model education advisors (these could be teachers that have a strong interest and acts as 'champions' and are seconded to this work) will work closely with teachers to fulfil the following; Initiate development and modification of educational games

- Suggest/allocate related learning theories

- Champion gamification ideas

- $\quad$ Supporting best practise

\section{CONCLUSION}

The recent unforeseen COVID-19 pandemic and consequent government lockdown directives and home schooling has created a new educational environment for pupils, parents and teachers. Some positive aspects of the new challenges have been increased commitment to community, willingness to embrace novel approaches to learning and teaching and increased interest in harnessing, sharing and developing novel approaches.

The use of gamification as a teaching tool is well known as a motivator. It can offer both an entertaining style and an easy and effective means of testing attainments. The current pause in conventional teaching has created an opportunity for stakeholders (parents, teachers and pupils) to exchange views via a chatroom incorporated in a repository environment which can then be assessed by educators and in conjunction with games developers and used to devise new games/teaching tools that can be shared and expanded across appropriate educational sectors with minimal financial support by government agencies. The whole movement can embrace a 'can-do attitude' to engage, motivate and entertain pupils. Figure 2 gives a visual representation outlining a proposed system.

In this paper, we suggest a synergistic digital hub to enhance a gamified learning environment to support all stakeholders in home schooling pathway during the COVID19 lockdown. However, the use of the collaboration platform could function as a learning support through normal circumstances of learning. The benefit of using gamification elements such as goals, social engagement, rewards, etc has been discussed in current published work. Therefore, the integration of expertise and experiences from teachers, parents/carers, game developers and education advisors altogether could provide a motivated and engaging learning experience for pupils. The intention of the dynamic repository would be to provide a 'sharing' resource to be used throughout the educational sector providing access to stakeholders to benefit the educational community. Future work will be to expand the model to develop a prototype web portal for application to the education system in Kingdom of Saudi Arabia.

\section{REFERENCES}

[1] C. Morillas Barrio, M. Munoz-Organero, and J. Sanchez Soriano, "Can Gamification Improve the Benefits of Student Response Systems in Learning? An Experimental Study," IEEE Trans. Emerg. Top. Comput., vol. 4, no. 3, pp. 429-438, Jul. 2016, doi: 10.1109/TETC.2015.2497459.

[2] Z. Fitz-Walter, D. Johnson, P. Wyeth, D. Tjondronegoro, and B. Scott-Parker, "Driven to drive? Investigating the effect of gamification on learner driver behavior, perceived motivation and user experience," Comput. Human Behav., vol. 71, pp. 586-595, Jun. 2017, doi: 10.1016/J.CHB.2016.08.050.

[3] BBC, "How to use Bitesize Daily - Our new service to help you learn at home-BBC Bitesize," 2020. https://www.bbc.co.uk/bitesize/articles/zfwrcqt (accessed Apr. 21, 2020).

[4] K. M. Kapp, The Gamification of Learning and Instruction. San Francisco: Pfeiffer, 2012.

[5] Y. Chou, Actionable Gamification: Beyond Points, Badges, and Leaderboard. Fermont: Octalysis Media, 2016.

[6] T. Bouzid, H. Darhmaoui, and F. Kaddari, "Promoting elementary mathematics learning through digital games," in Proceedings of the 2nd international Conference on Big Data, Cloud and Applications - BDCA'17, 2017, pp. 1-4, doi: 10.1145/3090354.3090451.

[7] E. D. Mekler, F. Brühlmann, A. N. Tuch, and K. Opwis, "Towards understanding the effects of individual gamification elements on intrinsic motivation and performance," Comput. Human Behav., vol. 71, pp. 525-534, Jun. 2017, doi: 10.1016/J.CHB.2015.08.048.

[8] F. Bellotti et al., "A Gamified Short Course for Promoting Entrepreneurship among ICT Engineering Students," in 2013 IEEE 13th International Conference on Advanced Learning Technologies, Jul. 2013, pp. 31-32, doi: 10.1109/ICALT.2013.14.

[9] A. Saggah, R. Campion, and A. Atkins, “An Agile Holistic Framework to Support Teachers in Pedagogical Gamification Design," in 2019 International Conference on Advances in the Emerging Computing Technologies (AECT), 2020, p. (In Press).

[10] BBC News, "Coronavirus: English teacher 'energised' by lockdown learning - BBC News," BBC News, Apr. 27, 2020. https:/www.bbc.co.uk/news/uk-england-bedsbucks-herts-52383295 (accessed Apr. 27, 2020).

[11] E. G. R. Flores, M. Soledad, R. Montoya, J. Mena, M. S. R. Montoya, and J. Mena, Challenge-based gamification and its impact in teaching mathematical modeling. New York, New York, USA: ACM Press, 2016, pp. 771-776. 
[12] A. Saggah, R. Campion, and C. Stanier, "An Investigation of the Role of the Teacher in Gamified Learning in Primary Schools," in EDULEARN18 Proceedings, 2018, pp. 7700-7708, Accessed: Sep. 25, 2018. [Online]. Available: https://library.iated.org/view/SAGGAH2018ANI.

[13] A. Saggah, R. Campion, and C. Stanier, "A Collaborative Gamification Design Framework in an Educational Context," in ICERI2018 Proceedings, 2018, pp. 2410-2414, doi: 10.21125/iceri.2018.

[14] J. Simone, A. Hauptman, and M. Hasty, "Better Together on Behalf of Our Children," Read. Teach., vol. 73, no. 3, pp. 281-289, Nov. 2019, doi: $10.1002 / \operatorname{trtr} .1825$.

[15] A. Gordillo, D. Gallego, E. Barra, and J. Quemada, "The city as a learning gamified platform," in 2013 IEEE Frontiers in Education Conference (FIE), Oct. 2013, pp. 372-378, doi: 10.1109/FIE.2013.6684850.

[16] S. Mystakidis, N. Lambropoulos, H. M. Fardoun, and D. M. Alghazzawi, "Playful Blended Digital Storytelling in 3D Immersive eLearning Environments," in Proceedings of the 2014 Workshop on Interaction Design in Educational Environments - IDEE '14, 2014, pp. 97-101, doi: $10.1145 / 2643604.2643632$.

[17] A. Botha and M. Herselman, "ICTs in Rural Education," in Proceedings of the 2015 Annual
Symposium on Computing for Development - DEV '15, 2015, pp. 105-113, doi: 10.1145/2830629.2830646.

[18] C. S. González et al., "Learning healthy lifestyles through active videogames, motor games and the gamification of educational activities," Comput. Human Behav., vol. 55, pp. 529-551, Feb. 2016, doi: 10.1016/J.CHB.2015.08.052.

[19] D. Kayımbaşığlu, B. Oktekin, and H. Hac1, "Integration of Gamification Technology in Education," Procedia Comput. Sci., vol. 102, pp. 668-676, Jan. 2016, doi: 10.1016/J.PROCS.2016.09.460.

[20] D. Kermek, D. Strmecki, M. Novak, and M. Kaniski, "Preparation of a hybrid e-learning course for gamification," in 2016 39th International Convention on Information and Communication Technology, Electronics and Microelectronics (MIPRO), May 2016, pp. 829-834, doi: 10.1109/MIPRO.2016.7522254.

[21] N. Özdener, "Gamification for enhancing Web 2.0 based educational activities: The case of pre-service grade school teachers using educational Wiki pages," Telemat. Informatics, Apr. 2017, doi: 10.1016/J.TELE.2017.04.003. 